\title{
Preparation and Property Study of CMCS Complexes
}

\author{
Lidan Dong ${ }^{1,2}$, Changping Wei ${ }^{1,}$, , Shuang Sun ${ }^{1}$, Fengming Wang ${ }^{1}$ \\ ${ }^{1}$ School of Materials Science and Engineering, Changchun University of Science and Technology, Changchun, China \\ ${ }^{2}$ School of Drug and Food, Changchun Medical College, Changchun, China
}

Email address:

573972433@qq.com (Lidan Dong), changpingwei@hotmail.com (Changping Wei)

${ }^{*}$ Corresponding author

\section{To cite this article:}

Lidan Dong, Changping Wei, Shuang Sun, Fengming Wang. Preparation and Property Study of CMCS Complexes. International Journal of Biomedical Materials Research. Vol. 5, No. 3, 2017, pp. 44-49. doi: 10.11648/j.ijbmr.20170503.13

Received: June 30, 2017; Accepted: July 15, 2017; Published: August 1, 2017

\begin{abstract}
To further enhance the carboxymethyl chitosan (collectively, carboxymethyl chitosan, CMCS) water-soluble and effect of coagulation and hemostatic, O-carboxymethyl chitosan (O-CMCS) and N,O-carboxymethyl chitosan (CMCS) are prepared by carboxymethyl chitosan modification under alkaline conditions, then Folic Acid-O-carboxymethyl chitosan (FA-O-CMCS) and Ca-nanocarboxymethyl chitosan(Ca-nmCMCS) are prepared by compounding folic acid and $\mathrm{Ca}^{2+}$, respectively. The samples are characterized by Infrared spectrum(IR), X-ray diffraction(XRD) and scanning electron microscope (SEM), and compared with each other, with the results showing as follows: FA-O-CMCS and Ca-nmCMCS are successfully synthesized respectively. Comparison of the two results shows that Ca-nmCMCS is more stable, and has smaller and more uniform particle size about $27.4 \mathrm{~nm}$, better dispersion without adhesion and stronger crystalline structure corresponding to the result of the solubility. But, the coagulation and hemostasis time of FA-O-CMCS is shorter than Ca-nmCMCS, and the former has the better effects of rapid coagulation and hemostasis. This provids a wide range of prospects for the application of Ca-nmCMCS and FA-O-CMCS.
\end{abstract}

Keywords: Carboxymethyl Chitosan, Carboxymethyl Chitosan Complexes, Complex Characterization, Performance

\section{Introduction}

Red blood cell membrane is composed of proteins, lipids, glycolipids and inorganic ions and so on, also, negatively charged in its surface. Red blood cell tends to adhere to the surface of the material positively charged [1]. Chitosan is the only natural alkaline polysaccharide positively charged [2], owning some advantages, such as, biodegradability and biocompatibility, etc $[3,4]$. Moreover, electric charge reaction with the amino and amino acid residues of the cell membrane surface, so that a large number of red blood cells get adhesion and aggregation in the wound, thrombosis, and the blood solidify [5]. However, chitosan forms chitosan sol because of being uniformly dispersed in water, and the viscosity is large, which limits chitosan' applications. The main mechanism of carboxymethylation of chitosan is that $\mathrm{N}, \mathrm{O}$-carboxymethyl chitosan is generated on amino and C6-OH simultaneously, while O-CMCS mainly is generated on C6-OH. But, carboxymethyl chitosan is still sparingly soluble, high viscosity. Carboxymethyl chitosan has better immunity, histocompatibility and biodegradability and promotes wound healing and so on [6-9]. In addition, O-carboxymethyl chitosan keeps a large number of reservations hemostatic group as amino. Calcium is a coagulation cofactor, and activiate the coagulation factors is involved in the process, which will help stop the bleeding. Folic acid-folate receptor targeted as a new drug is favored in recent years [10]. Folic acid possesses non-immunogenic and simple chemical properties. By the role of amino in ligand-receptor, gene and targeting drug is introduced into pathological tissues and cells, but normal cells are unaffected. In consequence, the safety and effectiveness of treatment are greatly increasing [11].

In this paper, nanometer CMCS (nmCMCS) was prepared by adopting the simplest method of synthesis and optimizing the experimental conditions, on the basis of summarizing the preparation methods of CMCS. In order to optimize the nmCMCS in hemostasis material performance, calcium ion which had better hemostatic effect evaluation on the modern medicine was selected. Then calcium ions was compounded 
with nmCMCS. To further expand the application in hemostatic medicine of CMCS, CMCS compounded withfolic acid-targeted drug. FA-O-CMCS which had better effect of coagulation and hemostatic was prepared. The structure and the related properties of carboxymethyl chitosan composites were tested.

\section{Experimental}

\subsection{Materials and Instruments}

Chitosan (BR), isopropyl alcohol, chloroacetic acid, calcium chloride, sodium tripolyphosphate, folic acid (BR), dimethyl sulfoxide, etc., and the rest are analytical grade.

\subsection{Preparation of $\mathrm{FA}-\mathrm{O}-\mathrm{CMCS}$}

Chitosan is dispersed in isopropanol and adds $\mathrm{NaOH}$ solution to this solution at room temperature. The $20 \mathrm{~mL}$ chloroacetic acid/ isopropanol solution is put into the mixture and the solution maintains at $40^{\circ} \mathrm{C}$ with stirring in water bath. O-CMCS is obtained through adjusted $\mathrm{PH}$ of about 7.0, washed, filtered and dried in vacuum drying oven at $60^{\circ} \mathrm{C}$. Folic acid is dissolved in $15 \mathrm{ml}$ dimethyl sulfoxide, then added an equimolar ratio of sodium laurate stirring for a period of time, in result of obtaining folic acid solution. A certain amount of O-CMCS is dissolved in acetic acid-sodium acetate buffer solution $(\mathrm{PH}=5.0)$ and then add dropwise folic acid solution. After reaction, the FA-O-CMCS presenting a pale yellow powder is prepared through centrifugation, washing and drying.

\subsection{Preparation of Ca-nmCMCS}

Chitosan was dissolved in a solution of isopropanol, added
$\mathrm{NaOH}$ solution into the solution, and basified for $2 \mathrm{~h}$. Thenthe solution of chloroacetic acid was added to the solution in water bath stirring for $3 \mathrm{~h}$. After the reaction, a pale yellow carboxymethyl group chitosan was prepared through adjusting $\mathrm{pH}$ to 7.0, washed, filtered and dried and stored in desiccators for further use. CMCS was dissolved isopropyl alcohol. After activation, sodium tripolyphosphate was added until a white opalescence state emerging. White powder of nano-carboxymethyl chitosan the reaction was obtained by filtering, washing, and drying. Add excess saturated $\mathrm{CaCl}_{2}$ solution to a solution containing $0.3 \mathrm{~g}$ of nano-carboxymethyl chitosan. After stirring in water bath, wash, filter and dry in result to obtain $\mathrm{Ca}-\mathrm{nmCMCS}$ appearing white powder.

\subsection{Charavterization and Performance}

At room temperature, compound's solubility is tested for coarse in $\mathrm{PH}=7$ aqueous; Fourier transform infrared spectroscopy is used to analysed samples at the measurement range of $4000-400 \mathrm{~cm}^{-1}$; X-ray diffraction spectroscopy is used to do structure analysis of the sample, and radiation source is $\mathrm{Cu} \mathrm{K \alpha}$ line, at the range of $10-90^{\circ}$; Surface morphology was scanned by electron microscope.

The adult male Kunming mice weighted between 18-26 g are selected in the test. Coagulation tests: Add potassium oxalate solution in a test tube. Then take the mouse eyes' blood $5 \mathrm{ml}$. Moreover, add the sample $(0.2 \mathrm{~g}$ sample/ $\mathrm{mL}$ of blood) to the test tube. At the same time observe and record the clotting time; Hemostasis test: Cut mice tail sharp at $3 \mathrm{~cm}$, and dress $0.2 \mathrm{~g}$ samples on the bleeding point. Then observe and record the time of stopping bleeding. That sets up the control group that the mice need the time in natural state in vitro coagulation and homeostasis. Parallel to three times each test and take the average.

\section{Results and Discussion}

\subsection{Solubility}

Table 1. Solubility test results.

\begin{tabular}{|c|c|c|c|c|}
\hline Quality & Quality dissolved(mg) & Solvent volume $(\mathrm{mL})$ & Solubility (mg/100mL) & solution state \\
\hline Chitosan & 0.006 & 20 & 0.03 & Sol, Viscous \\
\hline O-CMCS & 0.664 & 20 & 3.32 & Sol, Viscous \\
\hline FA-O-CMCS & 0.044 & 10 & 0.44 & Suspended on solvent \\
\hline CMCS & 0.511 & 20 & 3.935 & Sol, Viscous \\
\hline Ca-nmCMCS & 1.240 & 20 & 6.20 & Clear, Transparent \\
\hline
\end{tabular}

The results of each sample coarse solubility (solvent 100 mg per water) tests can be seen from table 1 . The solubility of chitosan, CMCS and O-CMCSpoor are all dispersed uniformly in aqueous solution like sol and more viscous, and it limits applications of chitosan and CMCS. FA-O-CMCS powder is low density, so that the powder can be suspended in the air and suspended upper the layer of aqueous solution. After stirring, its solubility is $0.44 \mathrm{mg}$. Ca-nmCMCS solution is clear and transparent and its solubility is $6.20 \mathrm{mg}$, which has much higher solubility than FA-O-CMCS, and the former is 14 times higher solubility than the latter. It broads the range of applications of chitosan and CMCS.

\subsection{Infrared Spectrum}

Figure 1 shows infrared spectrum of chitosan, CMCS and O-CMCS. -OH Stretching vibration peaks of chitosan appeared at $3334.69 \mathrm{~cm}^{-1}$. After different carboxymethylation modification, the $-\mathrm{OH}$ peaks of $\mathrm{N}$, O-CMCS shifted to $3332.76 \mathrm{~cm}^{-1}$, as O-CMCS shifted to $3294.19 \mathrm{~cm}^{-1}$ for $40 \mathrm{~cm}^{-1}$. Both shifts were red because that carboxymethyl cellulose is the electron-donating group. It indicates that $-\mathrm{CH}_{2} \mathrm{COOH}$ substitutes with $-\mathrm{OH}$ generating CMCS. After different carboxymethylation, C-O stretching 
vibration peak of chitosan at $1261.36 \mathrm{~cm}^{-1}$ disappeared. The peak at $1159.14 \mathrm{~cm}^{-1}$ is shifted to $1157.21 \mathrm{~cm}^{-1}$ of $\mathrm{N}$, O-CMCS and to $1108.99 \mathrm{~cm}^{-1}$ of O-CMCS. It describes that carboxymethyl substitutes $-\mathrm{OH}$ in chitosan in the process of modified chitosan. While that O-CMCS has higher degree offset level, this means the hydroxyl groups of O-CMCS has higher substitution ratio and larger the force between valence than CMCS. That $\mathrm{N}-\mathrm{H}$ characteristic peak of chitosan isat $3434.98 \mathrm{~cm}^{-1}$ and CMCS red shifted to $3433.06 \mathrm{~cm}^{-1}$, described carboxymethyl substituted $\mathrm{H}$ of chitosan amine. But, the peak of O-CMCS blue moves to $3444.19 \mathrm{~cm}^{-1}$ due to the formation of a sodium salt that is not replaced by electronic groups. At the same time, the chitosan C-N peak at $1326.93 \mathrm{~cm}^{-1}$ shiftes to $1323.08 \mathrm{~cm}^{-1}$, through carboxymethyl modified. But, the peak position didn't change in the process of O-carboxymethyl modified. As above further evidence O-CMCS amino groups don't react. In summary, chitosan hydroxyl and amine are substituted in the process of preparing CMCS. Differently, O-carboxymethyl reacts only on chitosan hydroxyl.

The IR spectrums the Ca-nmCMCS and FA-O-CMCS are presented in Figure 2. For Ca-nm CMCS, the hydroxyl stretching vibration peaks at $3332.76 \mathrm{~cm}^{-1}$ and $3130.25 \mathrm{~cm}^{-1}$ and $\mathrm{C}-\mathrm{O}$ stretching vibration peak at $1163.00 \mathrm{~cm}^{-1}$ shifted on nanocrosslinked, it is the result of the reaction between phosphate, $\mathrm{Ca}^{2+}$ and carboxyl, hydroxyl of CMCS. Ether bond stretching vibration peak areat $1091.63 \mathrm{~cm}^{-1}$ and $1249.79 \mathrm{~cm}^{-1}$. The former peak offsets, and the latter is a new peak due to reacting between the solvent structure electron-withdrawing and -O- key role. While the emergence of new peaks at $1660.60 \mathrm{~cm}^{-1}$ because of the impact of phosphate unsaturated structure cross-linked CMCS. After cross-linking folic acid, absorption peak of O-CMCS -OH at $3294.19 \mathrm{~cm}^{-1}$ disappeared. The peak at $3186.18 \mathrm{~cm}^{-1}$ blue moved to $3178.47 \mathrm{~cm}^{-1}$, and the $\mathrm{C}-\mathrm{O}$ absorption peak at $1108.99 \mathrm{~cm}^{-1}$ shifts to $1153.35 \mathrm{~cm}^{-1}$ due to de-OH reaction. Absorption peak of FA-O-CMCS - $\mathrm{NH}_{2}$ at $3425.23 \mathrm{~cm}^{-1}$ is shifted. C-NH $\mathrm{N}_{2}$ absorption at $1326.93 \mathrm{~cm}^{-1}$ unchanged and a new absorption peak at $1153.35 \mathrm{~cm}^{-1}$ peak appeared. It indicates amine de-H group occurs and the reaction adds the $\mathrm{C}-\mathrm{N}$ bond concentration in product. Some unsaturated absorption peak appeares in FA-O-CMCS due to the dehydration reaction mechanism of folic acid and O-CMCS is between carboxyl and amino.

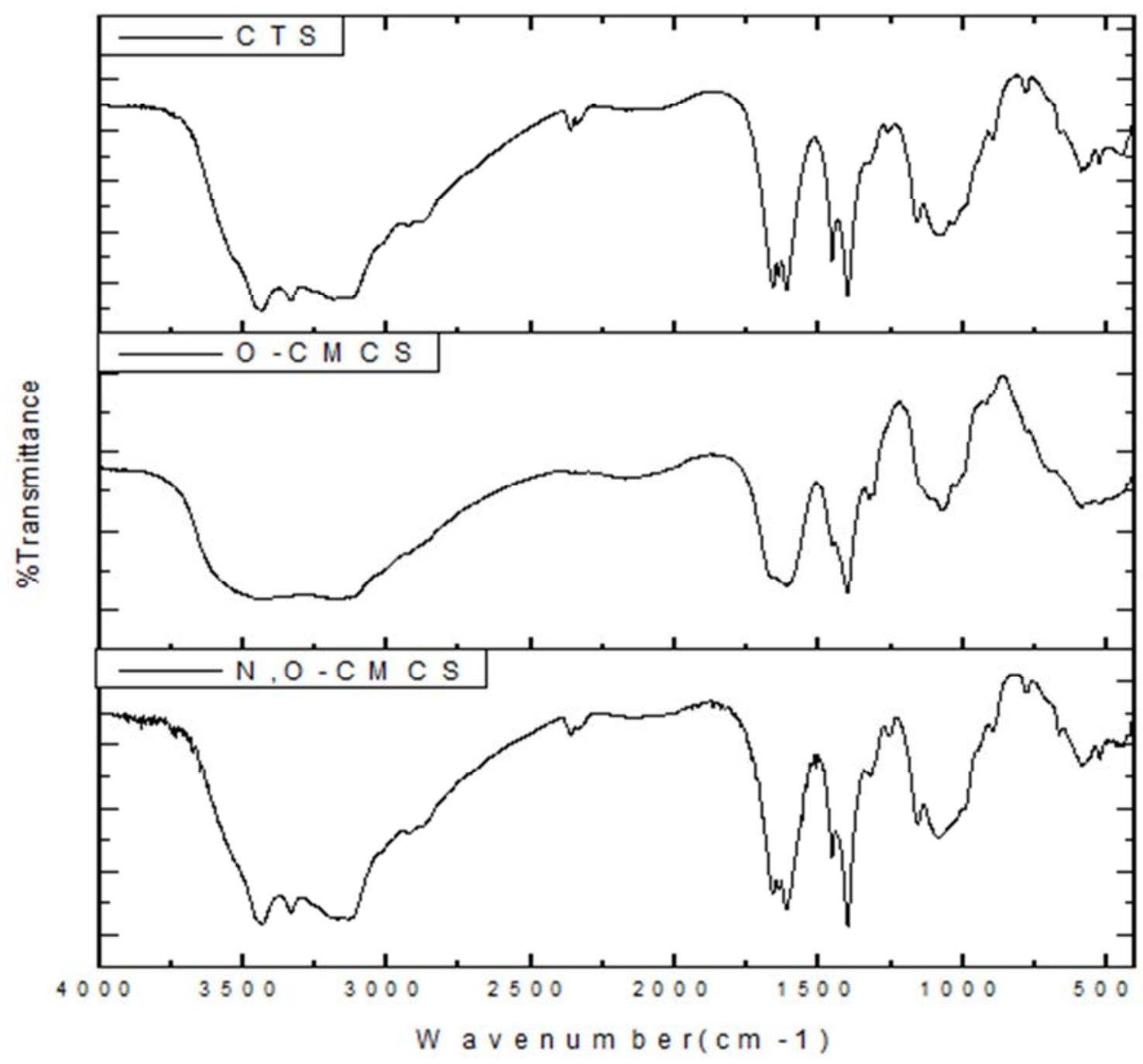

Figure 1. Infrared spectrum of CTS, CMCS O-CMCS. 


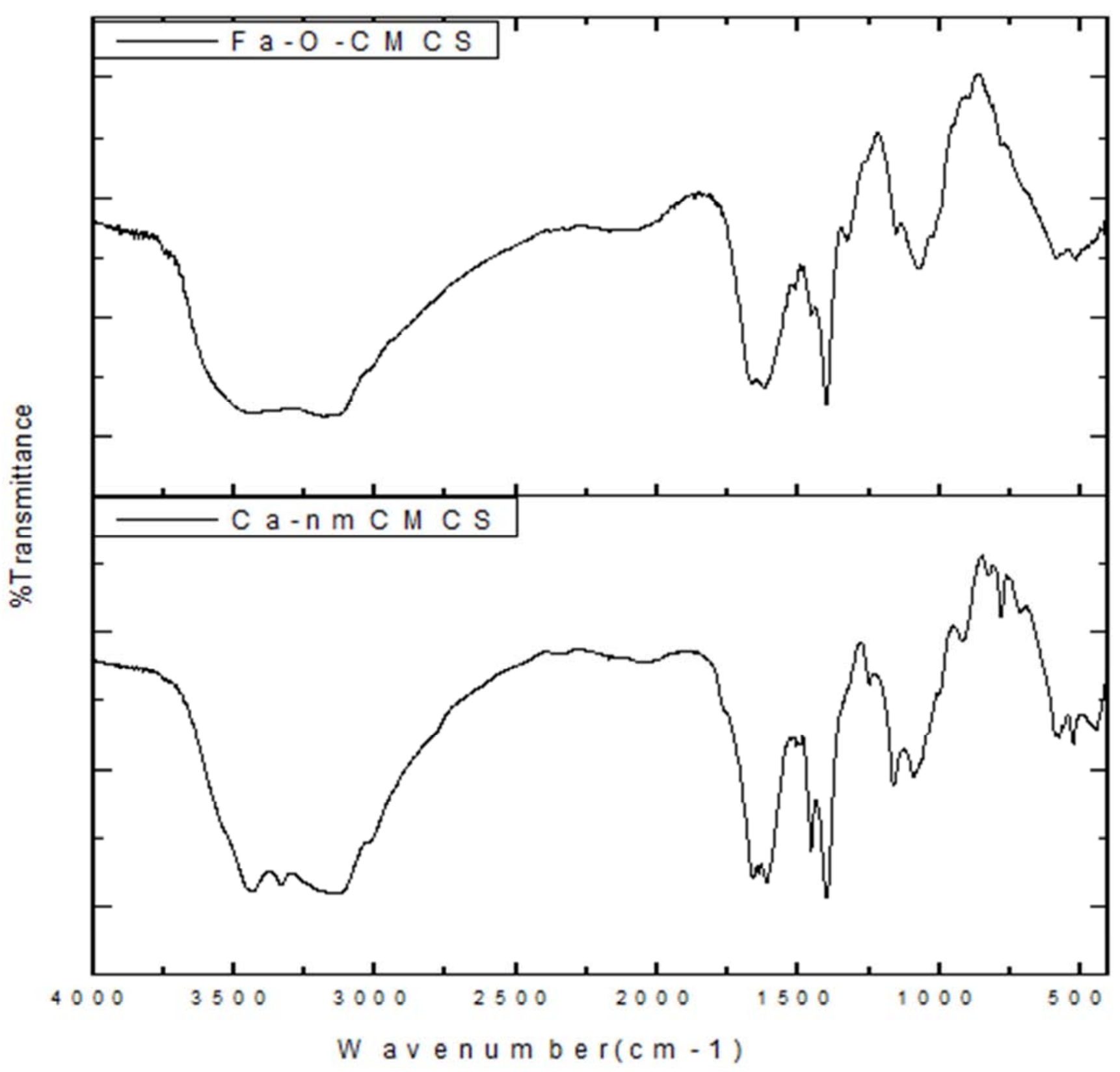

Figure 2. Infrared spectrum of FA-O-CMCS and Ca-nmCMCS.

\subsection{XRD Spectrum}

Figure 3 shows the XRD diffraction spectra of FA-O-CMCS and Ca-nmCMCS. The chitosan derivative peak intensity of $\mathrm{X}$-ray diffraction pattern proportional related to its degree of crystallinity. FA-O-CMCS diffraction peak appears at $21.1^{\circ}$ and $21.98^{\circ}$, these peaks partially overlapping. It was indicated that FA-O-CMCS complexes appeared crystalline and large forces among the group, and the composite structure was more stable. Strong diffraction peak of Ca-nmCMCS appears at $18.68^{\circ}, 19.22^{\circ}$ and $33.2^{\circ}$, and the former two peaks overlap. The peak is sharper than FA-O-CMCS. It indicates that crystalline of Ca-nmCMCS is stronger, which is inversely corresponding with both solubility.

\subsection{SEM Spectrum}

Scanning electron microscopy (SEM) images of FA-O-CMCS and Ca-nmCMCS are showed in Figure 4 and Figure 5. Both showed uniform spherical particles. Average particle size of FA-O-CMCS is about 0.9-1 $\mu \mathrm{m}$, buttle particle occured adhesion. Compared with it, Ca-nmCMCS looked better. Ca-nmCMCS is more uniform and its particle size of about $27.4 \mathrm{~nm}$ which was much smaller than FA-O-CMCS. It is because that Ca-nmCMCS experienced the nano and crosslinked with phosph and $\mathrm{Ca}^{2+}$ in this process which enforced in the bond. And the structure of Ca-nmCMCS was more stable. In addition, the single particles of Ca-nmCMCS was much clearer than FA-O-CMCS. Moreover, Ca-nmCMCS had no adhesion and 
with good distribution which was better morphology feature

Ca-nmCMCS are better than FA-O-CMCS. than the latter. Above all, the structure and morphology

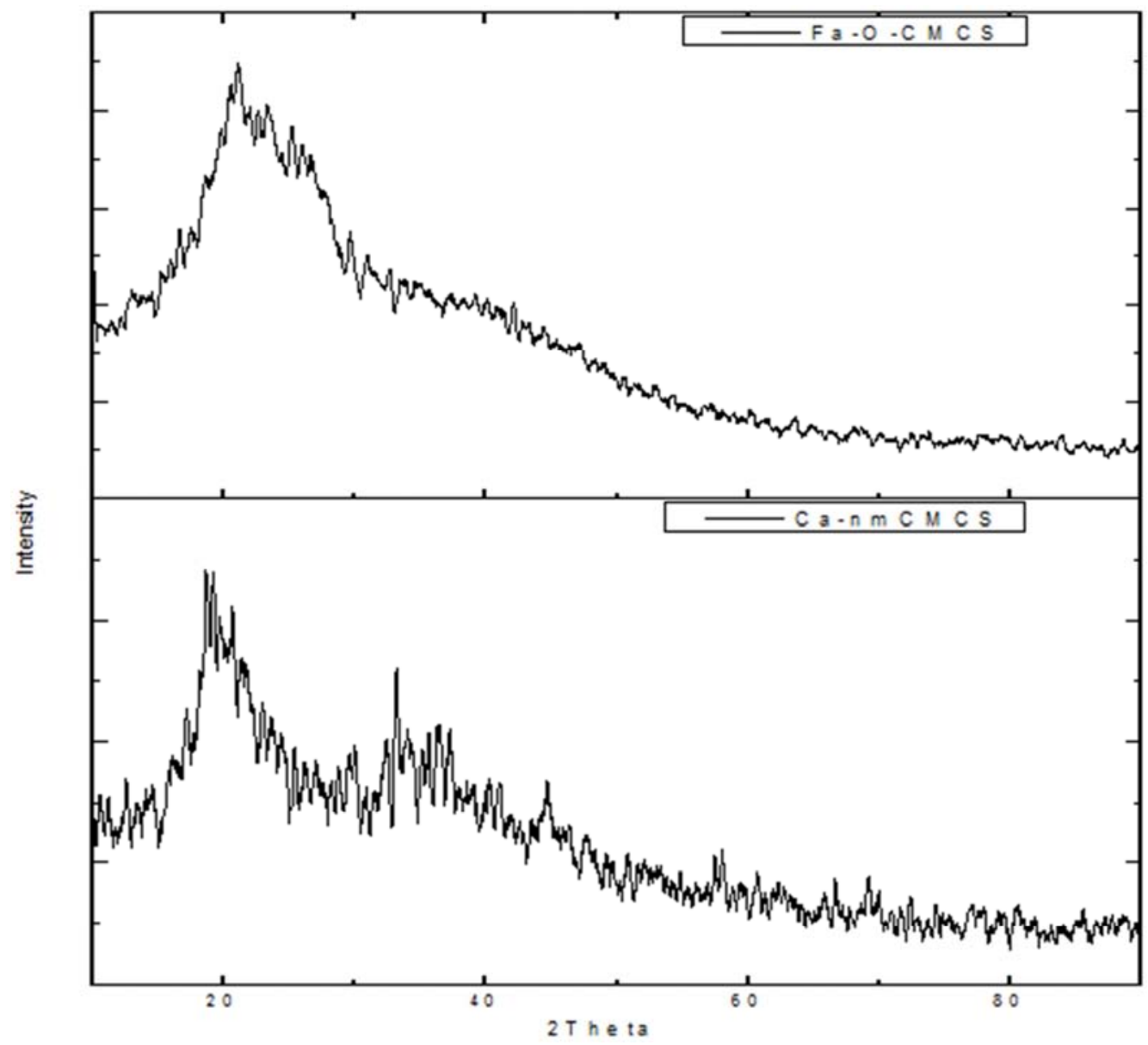

Figure 3. XRD spectrum of CTS, O-CMCS and FA-O-CMCS.

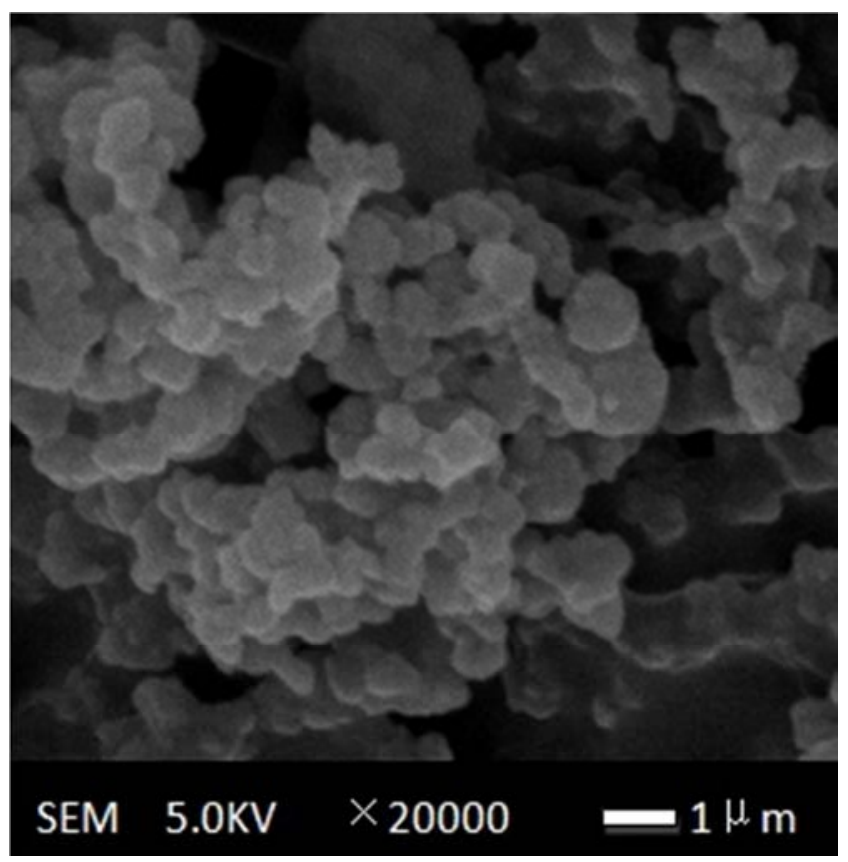

Figure 4. SEM spectrum of samples Fa-O-CMCS.

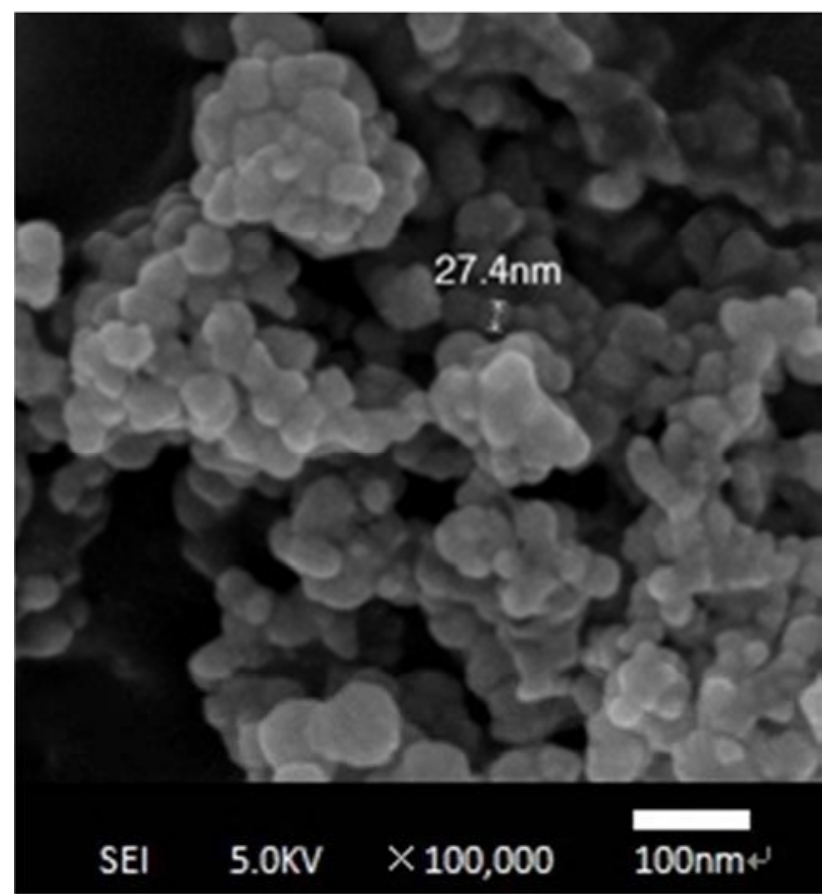

Figure 5. SEM spectrum of samples Ca-nmCMCS. 


\subsection{Performance Test}

Table 2. Clotting capability test of the sample.

\begin{tabular}{lllll}
\hline Sample name & $\mathbf{1}$ & $\mathbf{2}$ & $\mathbf{3}$ & average \\
\hline blank control group & $5^{\prime} 37^{\prime \prime}$ & $4^{\prime} 58^{\prime \prime}$ & $5^{\prime} 23^{\prime \prime}$ & $5^{\prime} 20^{\prime \prime}$ \\
CTS & $3^{\prime} 40^{\prime \prime}$ & $3^{\prime} 24^{\prime \prime}$ & $4^{\prime} 28^{\prime \prime}$ & $3^{\prime} 51^{\prime \prime}$ \\
CMCS & $3^{\prime} 50^{\prime \prime}$ & $2^{\prime} 38^{\prime \prime}$ & $3^{\prime} 45^{\prime \prime}$ & $3^{\prime} 24^{\prime \prime}$ \\
O-CMCS & $3^{\prime} 15^{\prime \prime}$ & $3^{\prime} 10^{\prime \prime}$ & $2^{\prime} 35^{\prime \prime}$ & $3^{\prime} 20^{\prime \prime}$ \\
FA-O-CMCS & $2^{\prime} 17^{\prime \prime}$ & $2^{\prime} 26^{\prime \prime}$ & $2^{\prime} 14^{\prime \prime}$ & $2^{\prime} 19^{\prime \prime}$ \\
nmCMCS & $3^{\prime} 10^{\prime \prime}$ & $3^{\prime} 32^{\prime \prime}$ & $2^{\prime} 35^{\prime \prime}$ & $3^{\prime} 06^{\prime \prime}$ \\
Ca-nmCMCS & $2^{\prime} 17^{\prime \prime}$ & $2^{\prime} 48^{\prime \prime}$ & $2^{\prime} 03^{\prime \prime}$ & $2^{\prime} 23^{\prime \prime}$ \\
\hline
\end{tabular}

Table 3. Hemostatic performance test of the sample.

\begin{tabular}{lllll}
\hline Sample name & $\mathbf{1}$ & $\mathbf{2}$ & $\mathbf{3}$ & average \\
\hline $\begin{array}{l}\text { blank control } \\
\text { group }\end{array}$ & $18^{\prime} 34^{\prime \prime}$ & $1^{\prime} 7^{\prime} 42^{\prime \prime}$ & $18^{\prime} 38^{\prime \prime}$ & $18^{\prime} 18^{\prime \prime}$ \\
CTS & $8^{\prime} 58^{\prime \prime}$ & $9^{\prime} 28^{\prime \prime}$ & $8^{\prime} 12^{\prime \prime}$ & $8^{\prime} 53^{\prime \prime}$ \\
CMCS & $7^{\prime} 24^{\prime \prime}$ & $7^{\prime} 18^{\prime \prime}$ & $7^{\prime} 44^{\prime \prime}$ & $7^{\prime} 29^{\prime \prime}$ \\
O-CMCS & $7^{\prime} 47^{\prime \prime}$ & $7^{\prime} 44^{\prime \prime}$ & $8^{\prime} 02^{\prime \prime}$ & $7^{\prime} 51^{\prime \prime}$ \\
FA-O-CMCS & $4^{\prime} 38^{\prime \prime}$ & $4^{\prime} 23^{\prime \prime}$ & $4^{\prime} 17^{\prime \prime}$ & $4^{\prime} 26^{\prime \prime}$ \\
nmCMCS & $6^{\prime} 39^{\prime \prime}$ & $6^{\prime} 20^{\prime \prime}$ & $6^{\prime} 16^{\prime \prime}$ & $6^{\prime} 25^{\prime \prime}$ \\
Ca-nmCMCS & $5^{\prime} 11^{\prime \prime}$ & $4^{\prime} 56^{\prime \prime}$ & $5^{\prime} 02^{\prime \prime}$ & $5^{\prime} 03^{\prime \prime}$ \\
\hline
\end{tabular}

The hemostatic performance test results of blank, chitosan, CMCS, O- CMCS, FA-O-CMCS and Ca-nmCMCS as shown in table 2, table 3 below. It is shown that chitosan can shorten significantly the hemostatic time. After modified carboxymethyl, CMCS hemostatic times shorten for $27^{\prime \prime}$ and 1 '24", as O-CMCS shortens for 31" and 1'02". FA-O-CMCS hemostatic time were 2'19" and 4'26", Ca-nmCMCS hemostatic times were $2^{\prime} 23^{\prime \prime}$ and 5' 03", which proved that these composites both had better effect on hemostatic. The test achieves purposes of rapid hemostasis. FA-O-CMCS has better effect to shorten hemostatic time than Ca-nmCMCS, about 4" and 37" due to complex with folic acid causing a significant increase amino which has a bleeding target the function and has better effect than calcium ions in hemostatic.

\section{Conclusions}

(1) IR, XRD and SEM proved that FA-O-CMCS and Ca-nmCMCS had been both prepared. Compared with each other, $\mathrm{Ca}$ - nano $\mathrm{CMCS}$ has better crystalline structure, and it is more stable, and the result corresponds with the solubility test; complexes both showed a spherical shape. Moreover, Ca-nmCMCS particle had better dispersion, no adhesion, and the particle size was smaller than FA-O-CMCS.

(2) samples' coagulation and hemostasis performance test results showed that these complexes can both shorten the coagulation and hemostasis time effectively. Comparing with each other, FA-O-CMCS's effect was better.

(3) Ca-nmCMCS has higher solubility, which provides a basis for wider application of chitosan and CMCS;
FA-O-CMCS has better effect of hemostatic and coagulation.

\section{Acknowledgements}

The author wishes to express thanks to Professors Wei Changping and Jia Mingjun for suggestions and for providing the research samples, and to LuoHuan and Yang Tao for their assistance with practical measurements.

\section{References}

[1] Cai S S, Liu Z H, Chen L L, et al. Folic acid - Preparation and chitosan conjugates to human red blood cells [J]. Spectroscopy Laboratory. 2012. 29(3): 1702-1707. (in Chinese).

[2] Cheng Q Y, Xia W S. Evaluation of chitosan hemostatic activity and Biological Safety [D]. JIANG SU: Jiangnan University. 2013. (in Chinese).

[3] Guo M M, Wu D, Liu H, et al. Hemostatic sponge and Healing Effect of O-CMCS hemostatic [J]. China Pharmacist. 2012, (11). (in Chinese).

[4] Lu B, Qian L Q, Zhang Z L. Chitosan hemostatic mechanism and application of research [J]. Health Research. 2010, 30(1): 55-58.

[5] Ma Feng, Lu Y F, Qin Y, et al. N, O- Preparation and characterization of carboxymethyl chitosan magnetic composite microspheres [J]. Materials Engineering. 2014, (8): 41-45. (in Chinese).

[6] Siriwat Tungtong, Siriporn Okonogi, Sombat Chowwanapoonpohn, et al. Solubility, viscosity and rheological properties of water-soluble chitosan derivatives [J]. Maejo International Journal of Science and Technology. 2012, 6(02): 315-322.

[7] Wang D S, Li J G, Li H P, et al. Preparation and drug releasing property of magnetic chitosan-5--fluorouracil nano-particles [J]. Transactions of Nonferrous Metals Society of China. 2009. 19(5): 1232-1236.

[8] Wang Z H, Jiang Y Y, Yu X H, et al. Effects of chitosan and its derivatives on the immune function in mice $[\mathrm{J}]$. Food Science. 2016. 37(01): 198-202. (in Chinese).

[9] WU R P, LI Q L, Liu X X, et al. Preparation and Characterization of folate-conjugated O-CMCS [J]. Technology and Development of Chemical Industry. 2013, 42(2): 1-3. (in Chinese).

[10] Yin G, Hou C L. Experimental study film thermosensitive chitosan hemostatic hemostatic effect [J]. Shanghai: Second Military Medical University. 2014. (in Chinese).

[11] Xing Z H, Fang G Z. Folic acid ionomer prepared folic acid-chitosan microsphere production [J]. Heilongjiang Medicine. /2011, 24(6): 915-917. (in Chinese). 